STOMACH

\title{
Stomach regulates energy balance via acylated ghrelin and desacyl ghrelin
}

\author{
A Asakawa, A Inui, M Fujimiya, R Sakamaki, N Shinfuku, Y Ueta, M M Meguid, M Kasuga
}

Gut 2005;54:18-24. doi: 10.1136/gut.2004.038737

See end of article for authors' affiliations

Correspondence to: Professor A Invi, Division of Diabetes, Digestive and kidney Diseases,

Department of Clinical Molecular Medicine, Kobe University Graduate

School of Medicine, 7-5-1

Kusunoki-cho, Chuo-ku, Kobe 650-0017, Japan; inui@med.kobe-u.ac.jp

Accepted for publication 14 June 2004
Background/Aims: The gastric peptide ghrelin, an endogenous ligand for growth-hormone secretagogue receptor, has two major molecular forms: acylated ghrelin and desacyl ghrelin. Acylated ghrelin induces a positive energy balance, while desacyl ghrelin has been reported to be devoid of any endocrine activities. The authors examined the effects of desacyl ghrelin on energy balance.

Methods: The authors measured food intake, gastric emptying, c-Fos expression in the hypothalamus, and gene expression of hypothalamic neuropeptides in mice after administration of desacyl ghrelin. To explore the effects of long term overexpression of desacyl ghrelin, transgenic mice that overexpressed desacyl ghrelin were created.

Results: Administration of desacyl ghrelin decreased food intake and gastric emptying rate through an action on the paraventricular nucleus and the arcuate nucleus in the hypothalamus. Gene expression of anorexigenic cocaine and amphetamine regulated transcript and urocortin in the hypothalamus was increased by desacyl ghrelin. Desacyl ghrelin overexpressing mice exhibited a decrease in body weight, food intake, and fat pad mass weight accompanied by moderately decreased linear growth. Gastric emptying was also decreased in desacyl ghrelin overexpressing mice.

Conclusions: These findings indicate that in contrast to acylated ghrelin, desacyl ghrelin induces a negative energy balance by decreasing food intake and delaying gastric emptying. The effect is mediated via the hypothalamus. Although derived from the same precursor, the inverse effects of these two peptides suggest that the stomach might be involved as an endocrine organ in the regulation of the energy balance.
G hrelin, a 28-amino acid peptide with structural resemblance to motilin, was recently identified in the stomach as an endogenous ligand for growth hormone secretagogue receptor (GHS-R). ${ }^{1}$ The ghrelin gene is predominantly expressed in the stomach and ghrelin is secreted into the circulatory system. Two major molecular forms of ghrelin are found in the stomach and plasma: acylated ghrelin, which has n-octanoylated serine in position 3; and desacyl ghrelin. ${ }^{2}$ Other minor forms of ghrelin are also present in the stomach and plasma. ${ }^{3}$ The n-octanoyl modification of ghrelin appears to be essential for $\mathrm{GH}$ releasing activity as endogenous ligand for GHS-R. ${ }^{1}$ Acylated ghrelin is involved in the regulation of GH secretion, energy balance, gastrointestinal motility, cardiac performance, and anxiety. ${ }^{4-8}$ Expression and secretion of ghrelin are increased by fasting and are reduced by feeding. ${ }^{45}$ In addition, diet induced weight loss increases plasma ghrelin level in humans. ${ }^{9}$ Administered acylated ghrelin induces body weight gain and adiposity by promoting food intake and decreasing fat use or energy expenditure. ${ }^{45}{ }^{10}$ On the other hand, desacyl ghrelin has been reported to be devoid of any endocrine activities. However, plasma desacyl ghrelin concentration accounts for more than $90 \%$ of total circulating ghrelin. ${ }^{21}$ We investigated the effects of desacyl ghrelin on energy balance.

\section{METHODS}

\section{Animal experiments}

We used male mice of the ddy strain (34-37 g, 8-9 weeks old; Japan Slc, Shizuoka, Japan). The mice were individually housed in a regulated environment $\left(22\right.$ (SEM 2) ${ }^{\circ} \mathrm{C}, 55$ (SEM 10) \% humidity, 12:12 hours light:dark cycle with light on at 7:00am). Food and water were available ad libitum except as otherwise indicated. The mice were used only once each in the experiment. All experiments were approved by our university animal care committee. Mouse desacyl ghrelin and mouse acylated ghrelin were purchased from Phoenix Pharmaceuticals, Inc (Belmont, CA, USA) and the Peptide Institute (Osaka, Japan), respectively. Just before administration, each drug was diluted in $4 \mu \mathrm{l}$ of artificial cerebrospinal fluid (ACSF) for intra-third cerebroventricular (ICV) injection or in $100 \mu \mathrm{l}$ physiological saline for intraperitoneal (IP) injection.

\section{ICV substance application}

For ICV injection, the mice were anaesthetised with sodium pentobarbital $(80-85 \mathrm{mg} / \mathrm{kg} \mathrm{IP})$ and placed in a stereotaxic instrument seven days before the experiments. A hole was made in each skull by using a needle inserted $0.9 \mathrm{~mm}$ lateral to the central suture and $0.9 \mathrm{~mm}$ posterior to the bregma. A 24 gauge cannula bevelled at one end over a distance of $3 \mathrm{~mm}$ was implanted into the third cerebral ventricle for ICV injection. The cannula was fixed to the skull with dental cement and capped with silicon without an obtruder. A 27 gauge injection insert was attached to a microsyringe by PE-20 tubing.

\section{Feeding tests}

Experiments were started at 10:00am. Before feeding tests, mice were deprived of food for 16 hours with free access to water, or were given free access to food and water. A

Abbreviations: ACSF, artificial cerebrospinal fluid; AGRP, agouti related protein; ARC, arcuate nucleus; CART, cocaine and amphetamine regulated transcript; CRF, corticotrophin releasing factor; FFA, free fatty acids; GHS-R, growth hormone secretagogue receptor; G3PDH, glyceraldehyde 3-phosphate dehydrogenase; ICV, intra-third cerebroventricular(ly); MCH, melanin concentrating hormone; NTS, nucleus tractus solitarius; NMU, neuromedin U; PBS, phosphate buffered saline; POMC, proopiomelanocortin; PVN, paraventricular nucleus; RT-PCR, reverse transcription polymerase chain reaction. 
standard diet (CLEA Japan Inc, Tokyo, Japan) was used. Food intake was measured by subtracting uneaten food from initially premeasured food at 20 minutes, one hour, and two hours after administration and checking for food spillage. Acylated ghrelin was administered intracerebroventricularly ( $1 \mathrm{nmol} /$ mouse). Desacyl ghrelin was administered intracerebroventricularly $(0.1-1 \mathrm{nmol} / \mathrm{mouse})$ or intraperitoneally (0.3-3 nmol/mouse).

\section{Gastric emptying}

Before the experiments in gastric emptying, mice were food deprived for 16 hours with free access to water. The fasted mice had free access to preweighed pellets for one hour; they (except desacyl ghrelin-overexpressing mice) were then administered intracerebroventricularly $(0.1-1 \mathrm{nmol} / \mathrm{mouse})$ or intraperitoneally $(0.3-3 \mathrm{nmol} /$ mouse $)$ with desacyl ghrelin. The mice were deprived of food again for two hours after administration. Food intake was measured by weighing uneaten pellets. Mice were killed by cervical dislocation three hours after the start of experiments. Immediately after, the stomach was exposed by laparotomy, quickly ligated at both the pylorus and cardia, then removed; then the dry content was weighed. Contents were dried by a vacuum freeze drying system (Model 77400, Labconco Corp, Kansas City, MO, USA). Gastric emptying was calculated according to the following formula: gastric emptying $(\%)=\{1$ - (dry weight of food recovered from the stomach/weight of food intake)\} $\times 100 .{ }^{12-14}$

\section{c-Fos immunohistochemistry}

On the day of the study, mice placed on ad libitum access to food and water were administered intraperitoneally with desacyl ghrelin ( $3 \mathrm{nmol} /$ mouse), acylated ghrelin ( $3 \mathrm{nmol} /$ mouse), or physiological saline. Food was removed immediately from the cages to prevent induction of c-Fos immunoreactivity resulting from feeding, Ninety minutes after administration, mice were anaesthetised with sodium pentobarbital $(80-85 \mathrm{mg} / \mathrm{kg}$ IP) and perfused with isotonic phosphate buffered saline (PBS) followed by $4 \%$ paraformaldehyde, $0.5 \%$ glutaraldehyde, and $0.2 \%$ picric acid in $0.1 \mathrm{M}$ phosphate buffer. Brains were removed and postfixed with $4 \%$ paraformaldehyde and $0.2 \%$ picric acid in $0.1 \mathrm{M}$ phosphate buffer. Brains were cut into $50 \mu \mathrm{m}$ thick coronal sections in a cryostat. Slices were incubated with rabbit polyclonal anti-c-Fos antibody (Oncogene Science, Cambridge, MA, USA) diluted 1:5000. Slices were then processed for biotinylated goat anti-rabbit IgG and avidin biotin peroxidase complex. Immunoreaction was performed with $0.01 \%$ 3,3'-diaminobenzidine, $1 \%$ ammonium nickel sulphate, and $0.0003 \% \mathrm{H}_{2} \mathrm{O}_{2}$ solution. Presence of c-Fos

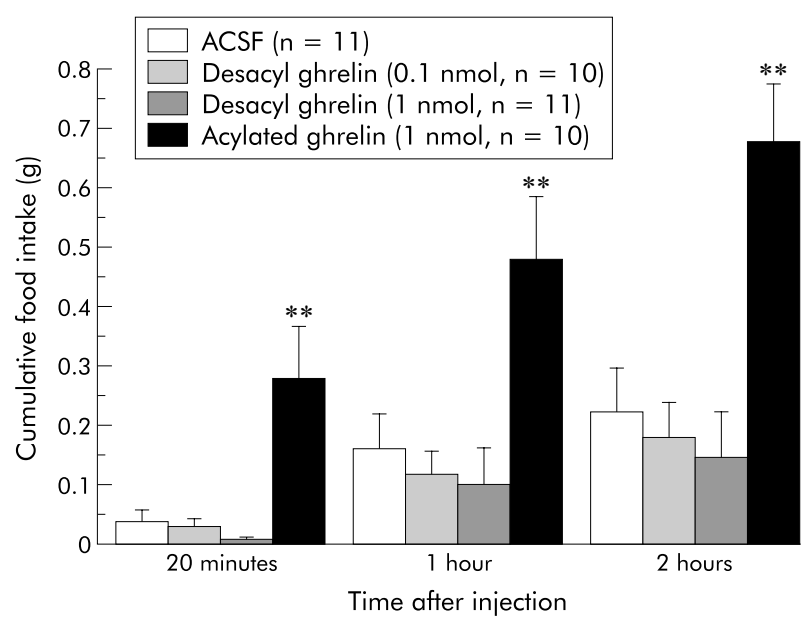

Figure 1 Effects of intracerebroventricularly administered desacyl ghrelin $(0.1-1 \mathrm{nmol} /$ mouse) and acylated ghrelin ( $1 \mathrm{nmol} /$ mouse) on cumulative food intake in non-food deprived mice. Results are expressed as mean (SEM). $n$ indicates the number of mice used. ${ }^{* *} p<0.01$ compared with the artificial cerebrospinal fluid (ACSF) treated control by Bonferroni's $t$ test.

immunoreactivity was revealed as a purple precipitate located in the neuronal nuclei. The exact anatomical localisation of c-Fos positive neurons in the brain nuclei was examined using cresyl violet staining, which was performed on the adjacent sections cut through each brain nucleus. The number of cells that showed c-Fos immunoreactivity was counted in paraventricular and arcuate nuclei.

\section{Real-time RT-PCR}

Mice were fasted for 12 hours. During the period of fasting, mice, except transgenic mice, were treated with desacyl ghrelin ( $3 \mathrm{nmol} / \mathrm{mouse}$ ) or physiological saline every six hours for 12 hours, with the third and final administration at 30 minutes before the mice were killed by cervical dislocation. Immediately after, the hypothalamic block was removed, frozen on dry ice, and stored at $-80^{\circ} \mathrm{C}$ until preparation of real-time reverse transcription polymerase chain reaction (real-time RT-PCR). Using the RNeasy Mini Kit (Qiagen KK, Tokyo, Japan) RNA was isolated from the hypothalamic block. Quantification of mRNA levels was performed with SYBR-green chemistry (Qiagen KK) using a one step RT-PCR reaction on an ABI PRISM 7700 Sequence Detection System purchased from Applied Biosystems Japan, Ltd. (Tokyo, Japan). The reaction was performed under

Table 1 Accession numbers and primers. G3PDH, glyceraldehyde 3-phosphate dehydrogenase

\begin{tabular}{llll}
\hline & $\begin{array}{l}\text { Accession } \\
\text { number }\end{array}$ & Forward & Reverse \\
\hline G3PDH & M32599 & ATGGTGAAGGTCGGTGTGAA & GAGTGGAGTCATACTGGAAC \\
NPY & NM_023456 & TTTCCAAGTTCCACCCTCATC & AGTGGTGGCATGCATTGGT \\
AGRP & NM_007427 & GAGTCCCAGGTCTAAGTCTGAATG & ATCTAGCACCTCCGCCAAAG \\
Orexin A & AF019566 & CGTAACTACCACCGCTTTAGCA & TGCCATTIACCAAGAGACTGACAG \\
MCH & AK020723 & GGAAGATACTGCAGAAAGATCCG & ATGAAACCGCTCTCGTCGTT \\
Beacon & NM_025401 & TTGCAACGACCGTCTCGGAAA & TTCAAGTCGCCGATGGTGTC \\
CART & NM_013732 & GCAGATCGAAGCGTGCAA & TTGGCCGTACTTCTTCTCGTAGA \\
POMC & NM_008895 & GGCTTGCAAACTCGACCTCT & TGACCCATGACGTACTCCG \\
NMU & NM_019515 & TGAAGCTTGTGCGTCCTTCT & AAAGCTCCCTCAGTGCAACAG \\
CRF & NM_205769 & CGCAGCCCTTGAATTCTTG & TCTGTTGAGATTCCCAGGC \\
Urocortin & NM_021290 & ACTGTCCATCGACCTCACCTTC & AAGGCTTTCGTGACCCCATA \\
\hline
\end{tabular}

NPY, neuropeptide Y; AGRP, agouti related protein; $M C H$, melanin concentrating hormone; CART, cocaine and amphetamine regulated transcript; POMC, proopiomelanocortin; NMU, neuromedin $\mathrm{U}$; CRF, corticotrophin releasing factor. 


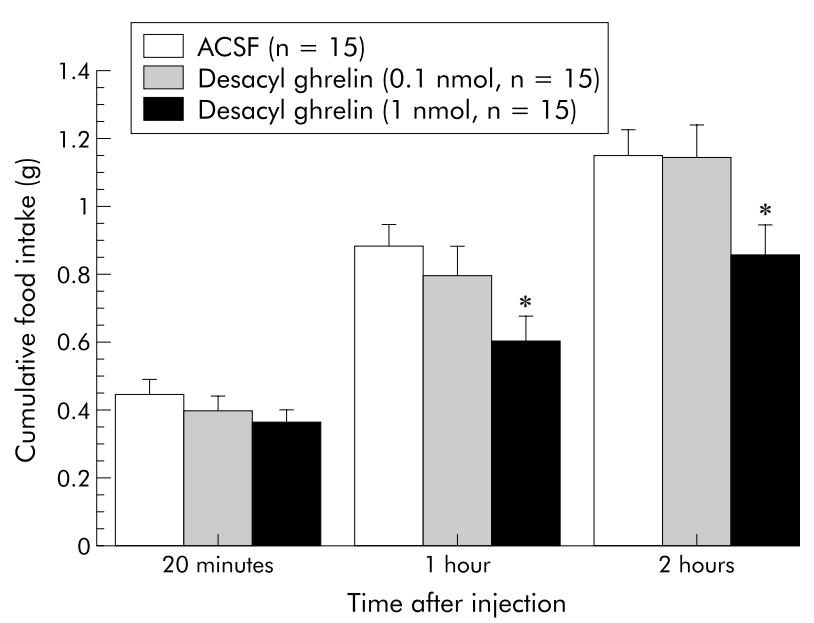

Figure 2 Inhibitory effects of intracerebroventricularly administered desacyl ghrelin $(0.1-1 \mathrm{nmol} / \mathrm{mouse})$ on cumulative food intake in 16 hour food deprived mice. Results are expressed as mean (SEM). $n$ indicates the number of mice used. ${ }^{*} p<0.05$ compared with the artificial cerebrospinal fluid (ACSF) treated control by Bonferroni's $t$ test.

standard conditions recommended by the manufacturer. We used the mouse glyceraldehyde 3-phosphate dehydrogenase $(\mathrm{G} 3 \mathrm{PDH})$ gene as an internal control. All expression data were normalised to G3PDH expression level from the same individual sample. The primers are shown in table 1 .

\section{Plasmid construction and production of transgenic mice}

Mouse ghrelin cDNA was obtained by RT-PCR from RNA isolated from mouse stomach. The sequence of the ghrelin cDNA was checked by ABI PRISM 3100 Genetic Analyzer purchased from Applied Biosystems Japan, Ltd (Tokyo, Japan). Plasmid pCAGGS ghrelin was constructed by inserting a mouse ghrelin cDNA into the unique EcoRI site between the CAG promoter and 3' flanking sequence of the rabbit $\beta$-globin gene of the pCAGGS expression vector. ${ }^{15}$ The pCAGGS expression vector was kindly donated by Professor Junichi Miyazaki (Osaka University, Osaka, Japan). The DNA fragment was excised from its plasmid by digestion with SalI and BamHI, then purified and microinjected into pronuclei of fertilised eggs obtained from BDFl female mice as reported previously. Transgenic (Tg) mice were usually identified by PCR and Southern blot analyses. Transgenic founders were mated with wild type C57/BL6 mice. The resultant Tg and non-Tg male littermates were used. Eight independent lines of $\mathrm{Tg}$ mice were identified with three to 12 copies of the ghrelin transgene. The mouse line (3-3) with the greatest ghrelin expression was used for all studies described in this paper. Selected studies were also performed with another line (11-1) containing 12 copies of the transgene to confirm observations made in the first line. All experiments were approved by the Kobe University Animal Care Committee.

\section{Southern blot analysis}

Mouse genomic DNA was isolated from mice tails. ${ }^{16}$ Mouse DNA was digested by EcoRI and PSTI, electrophoresed in 1\% agarose gel, then blotted onto nylon membranes. The DNA was probed with a ${ }^{32} \mathrm{P}$-labelled 2.93-kilobase salI-BamHI fragment of transgene. The blots were exposed onto a BAS-III imaging plate (Fuji Photo Film Co Ltd, Tokyo, Japan), and the transgene copy number was estimated by densitometry (Amersham Pharmacia Biotech AB, Uppsala, Sweden) relative to the $\beta$-actin-ghrelin standard.

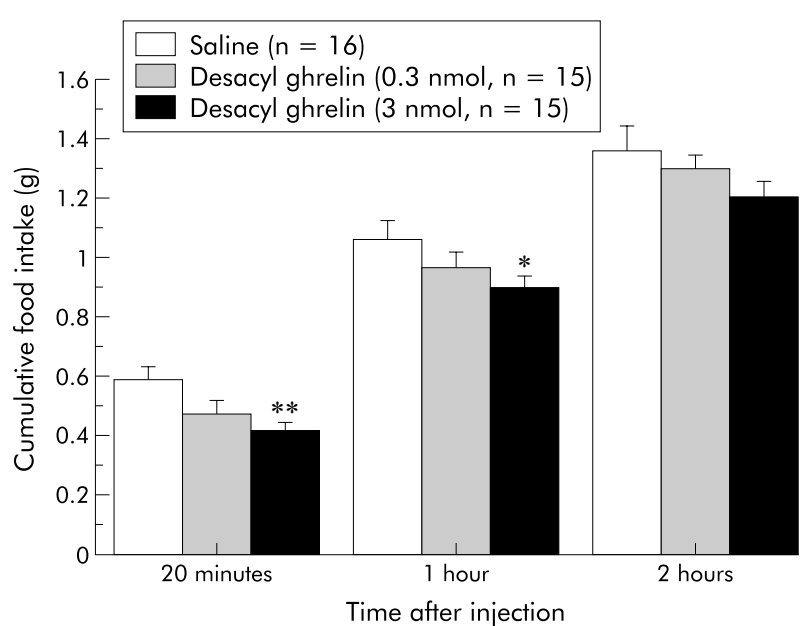

Figure 3 Inhibitory effects of intraperitoneally administered desacyl ghrelin (0.3-3 nmol/mouse) on cumulative food intake in 16 hour food deprived mice. Results are expressed as mean (SEM). $n$ indicates the number of mice used. ${ }^{*} \mathrm{p}<0.05 ;{ }^{* *} \mathrm{p}<0.01$ compared with saline treated control by Bonferroni's $t$ test.

\section{Northern blot analysis}

Total RNA was denatured with formaldehyde, electrophoresed in $1 \%$ agarose gel, then blotted onto a Hybond $\mathrm{N}^{+}$ membrane (Amersham Pharmacia Biotech AB, Uppsala, Sweden). Mouse ghrelin cDNA obtained by RT-PCR from RNA isolated from mouse stomach was labelled using a digoxigenin labelling kit (Roche Diagnostics KK, Tokyo, Japan). Membranes were hybridised with a digoxigenin labelled cDNA probe for ghrelin mRNA in the stomach, liver, and brain. Total integrated densities of hybridisation signals were determined by densitometry. Data were normalised to G3PDH mRNA abundance.

\section{Determination of body temperature}

A lubricated thermocouple was inserted $1.5 \mathrm{~cm}$ into the rectum of conscious mice. Rectal temperature was determined using a digital thermometer (Technol Seven Co. Ltd,

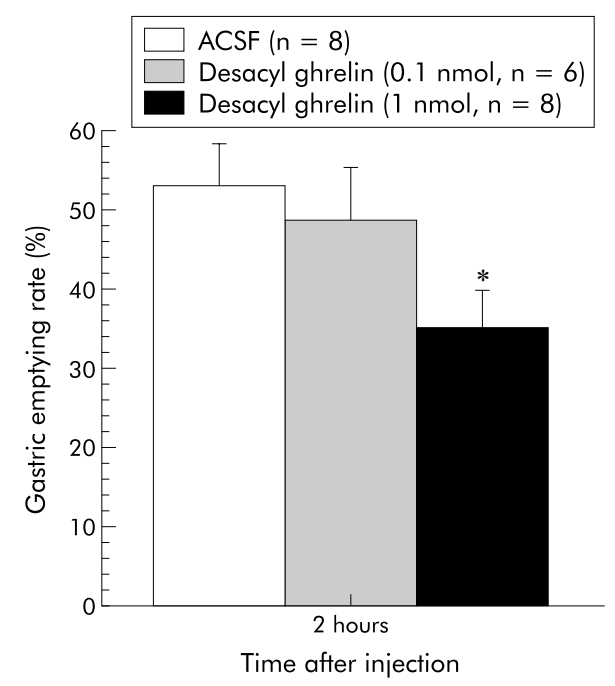

Figure 4 Inhibitory effects of intracerebroventricularly administered desacyl ghrelin (0.1-1 nmol/mouse) on gastric emptying rate two hours after administration. Results are expressed as mean (SEM). $n$ indicates the number of mice used. ${ }^{*} p<0.05$ compared with the artificial cerebrospinal fluid (ACSF) treated control by Bonferroni's $t$ test. 


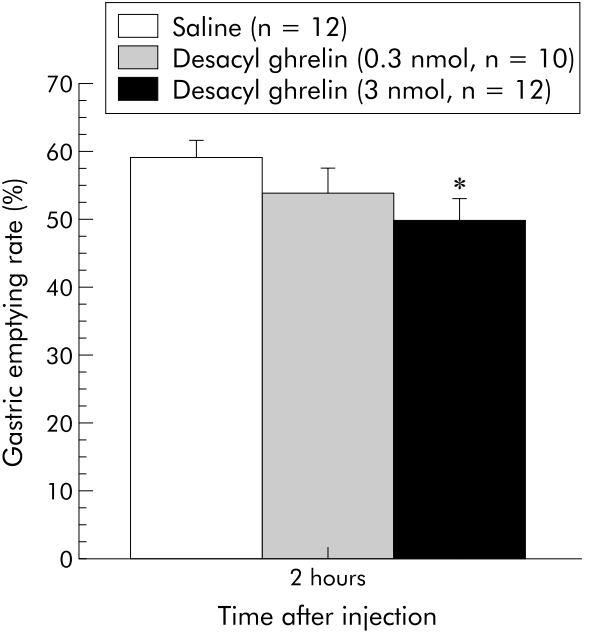

Figure 5 Inhibitory effects of intraperitoneally administered desacyl ghrelin (0.3-3 nmol/mouse) on gastric emptying rate two hours after administration. Results are expressed as mean (SEM). $n$ indicates the number of mice used. ${ }^{*} \mathrm{p}<0.05$ compared with saline treated control by Bonferroni's $t$ test.

Yokohama, Japan) in a room maintained at $22^{\circ} \mathrm{C}$ (SEM $\left.0.5^{\circ} \mathrm{C}\right)$.

\section{Oxygen consumption}

Oxygen consumption was determined by an $\mathrm{O}_{2} / \mathrm{CO}_{2}$ metabolism measuring system (Model MK-5000, Muromachikikai, Tokyo, Japan) at $22^{\circ} \mathrm{C}$. Chamber volume was $560 \mathrm{ml}$; airflow to the chamber was $500 \mathrm{ml} / \mathrm{min}$. Samples were taken every three minutes and a standard gas reference was taken every 30 minutes. Mice were kept unrestrained in the chamber without food or water during the light cycle; oxygen consumption was measured for two hours.

\section{Measurement of metabolic parameter and hormone concentrations}

Blood was obtained from the orbital sinus under ether anaesthesia. The entire sampling procedure was done in less than two minutes. Mice were killed by cervical dislocation. Immediately after the mice were killed, the epididymal fat pad mass was removed and weighed. Ghrelin concentrations were measured with RIA using polyclonal rabbit antibodies raised against the amino-terminal (amino acid positions 1 to 11 with O-n-octanoylation at Ser 3) or carboxyl-terminal fragments (amino acid positions 13 to 28) of ghrelin, as previously reported. ${ }^{17} 18$ Amino-terminal immunoreactivity represents acylated ghrelin alone (N IR), while carboxylterminal immunoreactivity corresponds to the sum of both acylated and desacyl ghrelin (C IR). Blood glucose was measured by the glucose oxidase method. Plasma insulin and free fatty acids (FFA) were measured by enzyme immunoassay and enzymatic method (Eiken Chemical Co Ltd, Tokyo, Japan), respectively. Triglycerides and total cholesterol were measured by enzymatic method (Wako Pure Chemical Inds Ltd, Tokyo, Japan). Growth hormone was measured with an immunoradiometric assay kit (Amersham Biosciences, Buckinghamshire, UK).

\section{Statistics}

Results are expressed as mean (SEM). Analysis of variance (ANOVA), followed by Bonferroni's $t$ test, was used to assess differences among groups. Changes in body weight and nose to anus length were compared using two-way ANOVA. A p value $<0.05$ was considered to be statistically significant.
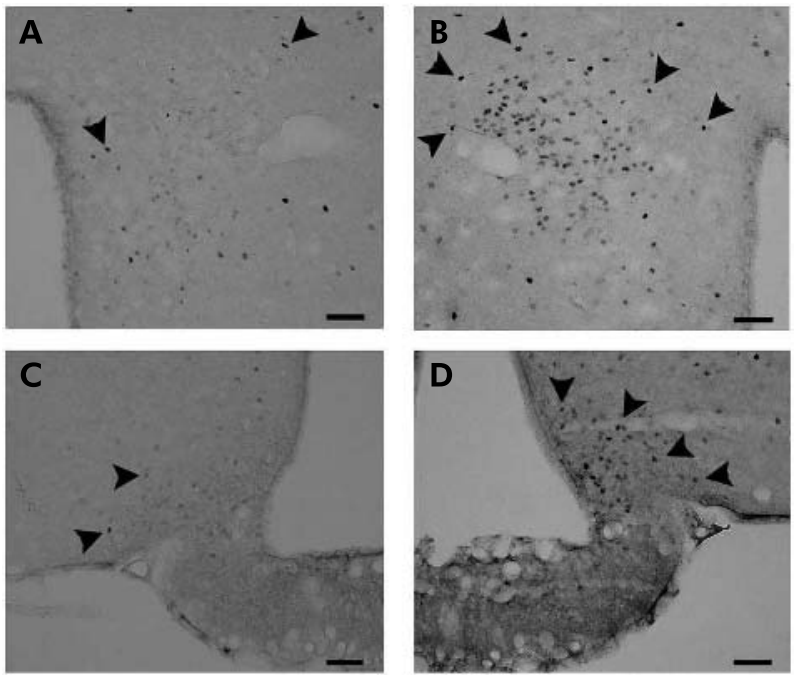

Figure 6 Effects of intraperitoneally administered desacyl ghrelin $(3 \mathrm{nmol} /$ mouse) on c-Fos expression in the hypothalamus 90 minutes after administration. (A) Paraventricular nucleus (PVN) of saline treated control. (B) PVN of desacyl ghrelin treated mice. (C) Arcuate nucleus (ARC) of saline treated control. (D) ARC of desacyl ghrelin treated mice. c-Fos positive neurons (arrows) were increased dramatically in desacyl ghrelin treated mice. Scale bars $=50 \mu \mathrm{m}$.

\section{RESULTS}

We investigated whether or not desacyl ghrelin influences feeding behaviour. We first administered desacyl ghrelin into the third cerebral ventricle in non-food deprived mice. Although acylated ghrelin potently increased food intake, desacyl ghrelin showed no increase, but a tendency to decrease food intake compared with ACSF treated control (fig 1). To evaluate the possibility that desacyl ghrelin has an anorexigenic activity, we examined the effects of desacyl ghrelin on food intake in food deprived mice. As shown in fig 2, centrally administered desacyl ghrelin significantly produced inhibitory effects on feeding behaviour. Moreover, we investigated whether intraperitoneally administered desacyl ghrelin has similar effects. Intraperitoneally as well

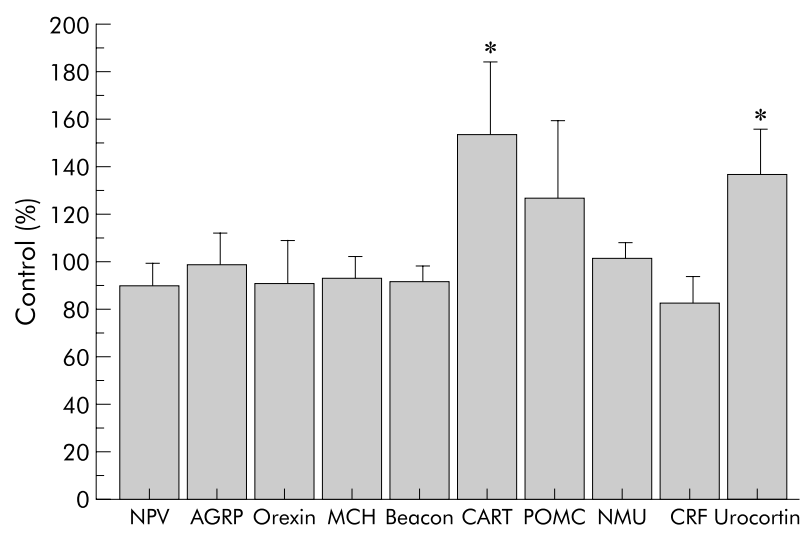

Figure 7 Effects of intraperitoneally administered desacyl ghrelin ( $3 \mathrm{nmol} /$ mouse every six hours for 12 hours) on hypothalamic peptides mRNA levels, as assessed by real-time reverse transcription polymerase chain reaction (real-time RT-PCR) in food deprived mice, expressed as a percentage of saline treated control. Results are expressed as mean (SEM) ( $n=5$ to 6 ). ${ }^{*} p<0.05$ compared with the saline treated control by Bonferroni's $t$ test. NPY, neuropeptide Y; AGRP, agouti related protein; $\mathrm{MCH}$, melanin concentrating hormone; CART, cocaine and amphetamine regulated transcript; $\mathrm{POMC}$, proopiomelanocortin; $\mathrm{NMU}$, neuromedin $\mathrm{U} ; \mathrm{CRH}$, corticotropin releasing hormone. 


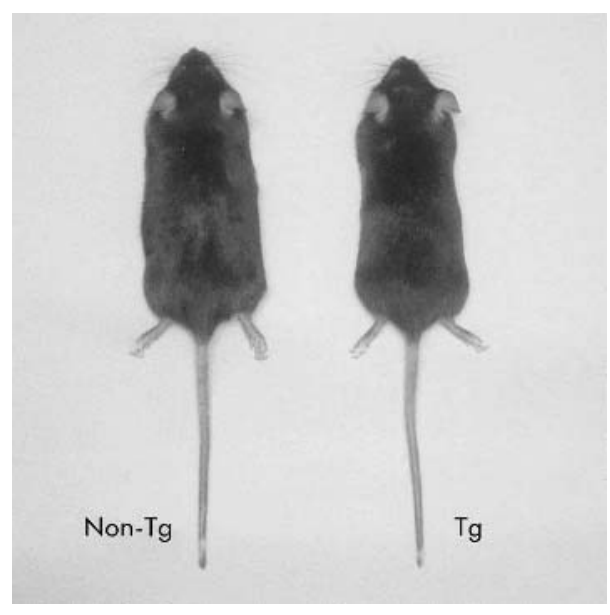

Figure 8 Gross appearance of transgenic $(\mathrm{Tg})$ mice overexpressing desacyl ghrelin and non-Tg littermates.

as intracerebroventricularly administered desacyl ghrelin significantly decreased food intake (fig 3). Although a primitive method was used to measure gastric emptying, intracerebroventricular administration of desacyl ghrelin ( $1 \mathrm{nmol} /$ mouse) reduced the rate of gastric emptying (fig 4). In addition, intraperitoneally administered desacyl ghrelin ( $3 \mathrm{nmol} /$ mouse) also produced a significant decrease in the gastric emptying rate (fig 5 ).

We next examined the effects of peripheral administration of desacyl ghrelin on c-Fos expression in the hypothalamus. Peripheral administration of desacyl ghrelin showed an increase in c-Fos expression in the paraventricular nucleus

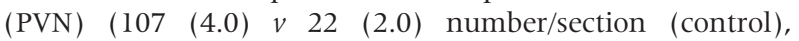
$\mathrm{p}<0.01)$ and in the arcuate nucleus (ARC) $(40(8.0) \vee 14.5$ (1.5) number/section (control), $\mathrm{p}<0.089$ ) (fig 6). Purple precipitates were distributed mainly in the medial parvocellular part of the PVN and in the ventrolateral part of the ARC. Peripheral administration of desacyl ghrelin showed no significant effects on c-Fos expression in the nucleus tractus solitarius (NTS) of the brainstem (4.0 (2.0) $v 2.5$ (0.5) number/section (control), p>0.54). Real-time RT-PCR analysis showed that desacyl ghrelin significantly increased anorexigenic CART and urocortin gene expression (fig 7). Inhibitory effect of desacyl ghrelin administered intraperitoneally ( $3 \mathrm{nmol} /$ mouse) on feeding was also observed at 90 minutes ( $1.04(0.03) v 1.19$ (0.05) g (control), p<0.05) after administration in 12 hour food deprived mice.

Transgenic mice were generated with overexpression of desacyl ghrelin under control of cytomegalovirus immediate early enhancer-chicken $\beta$-actin promoter (fig 8). Northern blot analysis revealed markedly increased ghrelin mRNA in stomach, brain, and liver tissues of Tg mice with 10 copies of the transgene (fig 9). Plasma desacyl ghrelin concentrations in $\mathrm{Tg}$ mice were elevated by approximately 30 -fold relative to non-Tg littermates (fig 10). No significant differences in plasma acylated ghrelin concentrations were noted between $\mathrm{Tg}$ mice and non-Tg littermates. As shown in table 2, body weights were significantly reduced in $\mathrm{Tg}$ mice with a tendency to decrease the linear growth (nose to anus length) $(p<0.097)$. Cumulative food intake and fat pad mass weight were also reduced in Tg mice (table 3). Despite the decreased body weight, hypothalamic gene expression of CART (161.7 (31.31) \% of control) and urocortin (153.4 (36.47) \% of control) showed a tendency to increase in 44 week old $\mathrm{Tg}$ mice, although this effect failed to reach statistical significance. Similar findings were observed in body weight ( 36.70 (1.248) v 39.86 (0.755) g (control), $\mathrm{p}<0.05)$, food intake

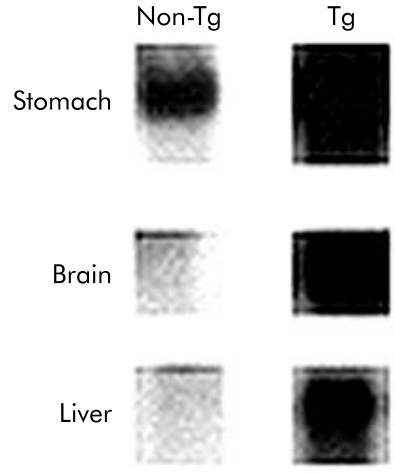

Figure 9 Ghrelin mRNA levels as assessed by Northern blot analysis in Tg mice.

(28.25 (1.248) v 31.07 (0.466) g/week (control), $\mathrm{p}<0.05)$, fat pad mass ( $1.346(0.158) v 1.723$ (0.088) g (control), $\mathrm{p}<0.05)$ and linear growth $(9.450 \quad(0.242) \quad v \quad 9.607 \quad(0.237) \mathrm{cm}$ (control)) of another line (11-1) of 44 week old Tg mice. Tg mice and non-Tg littermates exhibited no significant differences in body temperature. Oxygen consumption showed a tendency to increase in Tg mice (9.481 (0.759) $v$ $8.538(0.374) \mathrm{l} / \mathrm{h} / \mathrm{kg}$ (control)), although this effect failed to reach statistical significance. The plasma triglycerides level was significantly decreased with the accompanying tendency of the free fatty acids level to decrease moderately. There were no significant differences in plasma growth hormone
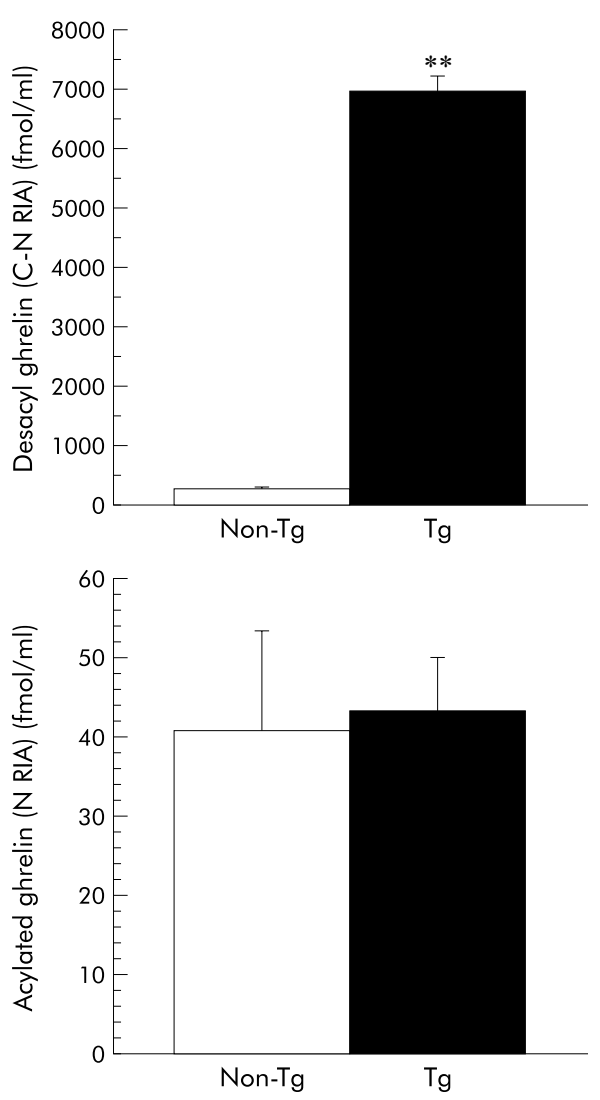

Figure 10 Plasma ghrelin concentrations in $\mathrm{Tg}$ mice and non- $\mathrm{Tg}$ littermates. Results are expressed as mean (SEM) $(n=3)$. ${ }^{* *} p<0.01$ compared with the non-Tg littermates by Bonferroni's $t$ test. This experiment was repeated in another line (1 1-1) of Tg mice, with similar results. 


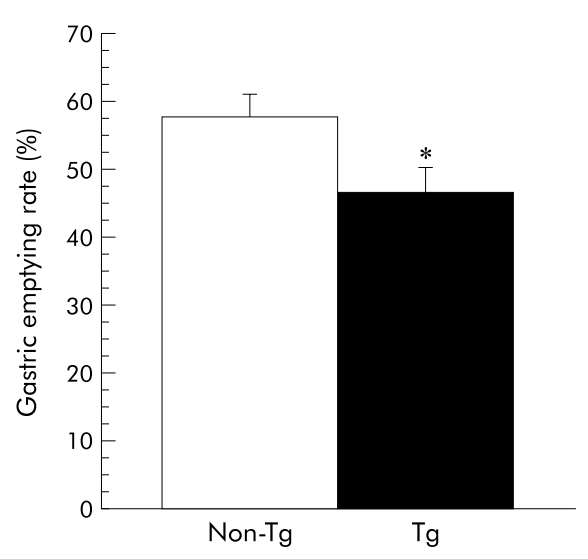

Figure 11 Gastric emptying rate in Tg mice and non-Tg littermates. Results are expressed as mean (SEM) $(n=10)$. ${ }^{*} p<0.05$ compared with the non-Tg littermates by Bonferroni's $t$ test.

levels. Gastric emptying rate in 44 week old $\mathrm{Tg}$ mice was decreased compared with the non-Tg littermates (fig 11).

\section{DISCUSSION}

In the past few years, much progress has been made in the understanding of how people maintain body weight and how homeostasis is affected. ${ }^{19-22}$ In 1999, ghrelin was discovered in the stomach as an appetite stimulatory signal from the periphery with structural resemblance to motilin. ${ }^{14523}$ Previous studies have shown that acylated ghrelin produces stimulatory effects on food intake via activation of NPY, AGRP, and orexin in the hypothalamus, and on gastric emptying. ${ }^{45-25}$ However, very little is known about the physiological role of desacyl ghrelin. We found that administration of desacyl ghrelin produced inhibitory effects on feeding. Notably, the anorexigenic effect of desacyl ghrelin was inversely proportioned to that of the orexigenic effect of acylated ghrelin. Considerable evidence cumulatively indicates that rapid gastric emptying is closely related to overeating and obesity, as delayed gastric emptying is to anorexia and cachexia. ${ }^{26-28}$ We previously reported that acylated ghrelin stimulates feeding behaviour with its mechanism of action involving the increase of gastric emptying. ${ }^{23}$ In the present study, administered desacyl ghrelin decreased the gastric emptying rate. Recently, in vitro desacyl ghrelin has been shown to inhibit cell proliferation in breast cancer cells and cell death in cardiomyocytes and endothelial cells through binding to an unknown receptor that is distinct from GHS-R. ${ }^{29}$ In addition, desacyl ghrelin influences contractility of the papillary muscle. ${ }^{30}$ These observations indicate that desacyl ghrelin is not devoid of any activities and may have an anorexigenic activity that is contrary to the orexigenic activity of acylated ghrelin.

Neuropeptides in the hypothalamus play a pivotal role in physiological mechanisms regulating food intake and body weight. ${ }^{19-22}$ Neuropeptides that stimulate feeding comprise NPY, AGRP, orexin, MCH, and beacon, whereas those that inhibit comprise CART, POMC, NMU, CRH, and urocortin. Previous studies have shown that acylated ghrelin and GHS-R antagonists influence feeding behaviour and gut motility. ${ }^{4-6} 3132$ GHS-R is present in various regions including the hypothalamus and intestine. ${ }^{145}$ In the hypothalamus, GHS-R is located in the ARC, where two orexigenic peptides, NPY and AGRP, are synthesised in the neuron. Up to now, acylated ghrelin has been reported to stimulate feeding behaviour with its mechanism of action involving activation of hypothalamic neurons. ${ }^{45-25}$ In the present study (unpublished data) and in previous reports, ${ }^{33}$ C-Fos expression in the
Table 2 Changes in body weight and nose to anus length with age in desacyl ghrelin transgenic $(\mathrm{Tg})$ mice and non-Tg littermates

\begin{tabular}{|c|c|c|c|c|}
\hline \multirow{2}{*}{$\begin{array}{l}\text { Age } \\
\text { (weeks) }\end{array}$} & \multicolumn{2}{|c|}{ Body weight (g) } & \multicolumn{2}{|c|}{ Nose to anus length $(\mathrm{cm})$} \\
\hline & Non-Tg & $\mathrm{Tg}$ & Non-Tg & $\mathrm{Tg}$ \\
\hline 11 & $26.93(0.258)$ & $26.38(0.649)$ & $8.318(0.083)$ & $8.225(0.095)$ \\
\hline 22 & $30.05(0.365)$ & $29.43(0.704)$ & $8.705(0.081)$ & $8.650(0.067)$ \\
\hline 44 & $37.69(1.673)$ & $33.90(0.631)^{*}$ & $9.594(0.216)$ & $9.175(0.124)$ \\
\hline \multicolumn{5}{|c|}{$\begin{array}{l}\text { Results are expressed as mean (SEM) ( } n=8 \text { to } 11) \text {. }{ }^{*} p<0.05 \text { compared } \\
\text { with the non-Tg littermates by two way analysis of variance (ANOVA) } \\
\text { followed by Bonferroni's } t \text { test. }\end{array}$} \\
\hline
\end{tabular}

ARC and the PVN was increased by administered acylated ghrelin. On the other hand, peripheral administration of desacyl ghrelin also showed an increase in c-Fos expression in the ARC and in the PVN. Gene expression of anorexigenic CART and urocortin in the hypothalamus was increased by administration of desacyl ghrelin. Both CART expressing neurons and urocortin expressing neurons are present in several brain regions that include the hypothalamus. ${ }^{34} 35$ Several studies have shown that CART and urocortin are endogenous satiety factors in the central nervous system. ${ }^{19-22}$

Date et al recently reported that GHS-R is synthesised in vagal afferent neurons in the nodose ganglion and transported to their afferent terminals in the stomach. ${ }^{36}$ It has been demonstrated that desacyl ghrelin has no effects on vagal nerve activities, even though acylated ghrelin acts as an orexigenic signal from periphery to the hypothalamus through the vagal nerve. ${ }^{23}{ }^{36}$ We also found that peripherally administered desacyl ghrelin showed no significant effects on c-Fos expression in the NTS of the brainstem. Banks et al recently reported that desacyl ghrelin can cross the bloodbrain barrier in the blood to brain direction, although acylated ghrelin enters the brain to a far lesser degree. ${ }^{37}$ Taken together, these observations indicate that desacyl ghrelin can act, on hypothalamic sites that are accessible to peripheral hormones, as a feeding suppressor through the endocrine pathway. On the other hand, the receptor for desacyl ghrelin has not been identified. However, our results indicate that desacyl ghrelin decreases food intake with its mechanism of action involving the hypothalamus and stomach. In addition, the inhibitory effect of desacyl ghrelin administered intraperitoneally on feeding was faster than that of desacyl ghrelin administered intracerebroventricularly, suggesting an involvement of peripheral mechanism(s) that are yet to be examined.

Table 3 Comparison of food intake, body temperature, epididymal fat mass, metabolic parameters, and growth hormone concentration in 44 week old desacyl ghrelin transgenic $(\mathrm{Tg})$ mice and non-Tg littermates

\begin{tabular}{lll}
\hline & Non-Tg & Tg \\
\hline Food intake (g/week) & $29.18(0.504)$ & $27.58(0.478)^{*}$ \\
Body temperature ( $\mathrm{C})$ & $36.13(0.158)$ & $36.30(0.103)$ \\
Fat pad mass $(\mathrm{g})$ & $1.413(0.154)$ & $1.063(0.075)^{*}$ \\
Glucose $(\mathrm{mmol} / \mathrm{I})$ & $8.680(0.366)$ & $8.066(0.233)$ \\
Insulin $(\mathrm{pmol} / \mathrm{I})$ & $558.8(146.7)$ & $430.5(50.80)$ \\
Cholesterol (mmol/l) & $1.949(0.145)$ & $2.030(0.072)$ \\
Triglycerides (mmol/I) & $0.462(0.089)$ & $0.209(0.032)^{*}$ \\
Free fatty acids (meq/l) & $1.873(0.161)$ & $1.575(0.098)$ \\
Growth hormone ( $\mu \mathrm{g} / \mathrm{l})$ & $3.425(0.357)$ & $3.280(0.436)$ \\
\hline
\end{tabular}

Mice were sacrificed four hours after removal of food. Results are expressed as mean (SEM) ( $n=8$ to 10). ${ }^{*} \mathrm{p}<0.05$ compared with the nonTg littermates by Bonferroni's $t$ test. 
To explore the effects of long term overexpression of desacyl ghrelin, we created Tg mice that overexpress desacyl ghrelin. As expected, the Tg mice gained less weight with reduced fat mass compared with non-Tg littermates. The linear growth showed a tendency to decrease in $\mathrm{Tg}$ mice. Consistent with the observation that administration of desacyl ghrelin decreased food intake and gastric emptying, these were reduced in $\mathrm{Tg}$ mice. There was no significant difference between Tg mice and non-Tg littermates in body temperature and oxygen consumption. Therefore, this thin phenotype of the $\mathrm{Tg}$ mice may be due, at least in part, to a decrease in food intake.

These findings indicate that desacyl ghrelin induces a state of negative energy balance and body weight decrease by inhibiting food intake in an inverse manner to acylated ghrelin. The effect of ghrelin on adipogenesis still remains to be determined. In bone marrow, Thompson et al recently reported that acylated ghrelin and desacyl ghrelin stimulate tibial bone marrow adipogenesis via a receptor other than GHS-R. ${ }^{38}$ On the other hand, Zhang et al reported that ghrelin inhibits adipogenesis in 3T3-Ll cell line by stimulation of preadipocyte cell proliferation via a novel ghrelin receptor subtype. ${ }^{39}$ The present study shows that effects of administered desacyl ghrelin on food intake and gastric emptying were contrary to those of acylated ghrelin. Moreover, desacyl ghrelin overexpressing mice showed thin phenotype with decreased food intake and gastric emptying rate. Therefore, the distinction between acylated ghrelin and desacyl ghrelin may be needed to investigate physiological and pathological functions of ghrelin. Previous studies have shown that plasma ghrelin concentration is increased by fasting and decreased by feeding. ${ }^{4}{ }^{17}$ Recently, Ariyasu et al reported that $70 \%$ food restriction decreases the ratio of desacyl ghrelin to acylated ghrelin in food restricted mice compared with ad libitum fed mice. ${ }^{18}$

Thus, the stomach may regulate energy balance via acylated ghrelin and desacyl ghrelin as an endocrine organ. Eating abnormalities are associated with various diseases including obesity, diabetes, anorexia nervosa, and cachexia. Better understanding of the role of ghrelin peptides may provide an entirely new therapeutic approach for treatment of these various diseases, which have become increasingly prevalent throughout the world.

\section{ACKNOWLEDGEMENTS}

The authors thank Dr Hiroshi Hosoda and Professor Kenji Kangawa (National Cardiovascular Center Research Institute, Osaka, Japan) for measurement of ghrelin concentration, Toshihiro Kaga and Miwako Katagi (Kobe University, Kobe, Japan) for technical assistance, and Professor Toshifumi Sakai (Saitama University, Saitama, Japan) for discussion.

\section{Authors' affiliations}

A Asakawa, A Inui, M Kasuga, Division of Diabetes, Digestive and Kidney Diseases, Department of Clinical Molecular Medicine, Kobe University Graduate School of Medicine, Kobe, Japan

M Fujimiya, Department of Anatomy, Shiga University of Medical Science, Shiga, Japan

R Sakamaki, N Shinfuku, International Center for Medical Research, Kobe University Graduate School of Medicine, Kobe, Japan Y Ueta, Department of Physiology, School of Medicine, University of Occupational and Environmental Health, Kitakyushu, Japan M M Meguid, Department of Surgery, SUNY Upstate Medical University, Syracuse, NY, USA

\section{REFERENCES}

1 Kojima M, Hosoda H, Date Y, et al. Ghrelin is a growth-hormone-releasing acylated peptide from stomach. Nature 1999;402:656-60.

2 Hosoda H, Kojima M, Matsuo H, et al. Ghrelin and des-acyl ghrelin: two major forms of rat ghrelin peptide in gastrointestinal tissue. Biochem Biophys Res Commun 2000;279:909-13.
3 Hosoda $\mathbf{H}$, Kojima M, Mizushima T, et al. Structural divergence of human ghrelin. Identification of multiple ghrelin-derived molecules produced by posttranslational processing. J Biol Chem 2003;278:64-70.

4 Invi A. Ghrelin: an orexigenic and somatotrophic signal from the stomach. Nat Rev Neurosci 2001;2:551-60.

5 Kojima M, Kangawa K. Ghrelin, an orexigenic signaling molecule from the gastrointestinal tract. Curr Opin Pharmacol 2002;2:665-8.

6 Fujino K, Inui A, Asakawa A, et al. Ghrelin induces fasted motor activity of the gastrointestinal tract in conscious fed rats. J Physiol 2003;550:227-40.

7 Nagaya N, Kangawa K. Ghrelin improves left ventricular dysfunction and cardiac cachexia in heart failure. Curr Opin Pharmacol 2003;3:146-51.

8 Carlini VP, Monzon ME, Varas MM, et al. Ghrelin increases anxiety-like behavior and memory retention in rats. Biochem Biophys Res Commun 2002;299:739-43.

9 Cummings DE, Weigle DS, Frayo RS, et al. Plasma ghrelin levels after dietinduced weight loss or gastric bypass surgery. N Engl J Med 2002;346: 1623-30

10 Tschop M, Smiley DL, Heiman ML. Ghrelin induces adiposity in rodents. Nature 2000;407:908-13.

11 Yoshimoto A, Mori K, Sugawara A, et al. Plasma ghrelin and desacyl ghrelin concentrations in renal failure. J Am Soc Nephrol 2002;13:2748-52.

12 Barrachina MD, Martinez V, Wang L, et al. Synergistic interaction between leptin and cholecystokinin to reduce short-term food intake in lean mice. Proc Natl Acad Sci U S A 1997:94:10455-60.

13 Asakawa A, Inui A, Yuzuriha $\mathrm{H}$, et al. Characterization of the effects of pancreatic polypeptide in the regulation of energy balance. Gastroenterology 2003; 124:1325-36

14 Roh J, Chang CL, Bhalla A, et al. Intermedin is a calcitonin/calcitonin generelated peptide family peptide acting through the calcitonin receptor-like receptor/receptor activity-modifying protein receptor complexes. J Biol Chem 2004;279:7264-74.

15 Niwa H, Yamamura K, Miyazaki J. Efficient selection for high-expression transfectants with a novel eukaryotic vector. Gene 1991;108:193-9.

16 Southern EM, Mitchell AR. Chromatography of nucleic acid digests on thin layers of cellulose impregnated with polyethyleneimine. Biochem J $1971 ; 123: 613-17$.

17 Ariyasu H, Takaya K, Tagami T, et al. Stomach is a major source of circulating ghrelin, and feeding state determines plasma ghrelin-like immunoreactivity levels in humans. J Clin Endocrinol Metab 2001;86:4753-8.

18 Ariyasu H, Takaya K, Hosoda H, et al. Delayed short-term secretory regulation of ghrelin in obese animals: evidenced by a specific RIA for the active form of ghrelin. Endocrinology 2002;143:3341-50.

19 Invi A. Transgenic approach to the study of body weight regulation. Pharmacol Rev 2000;52:35-61.

20 Spiegelman BM, Flier JS. Obesity and the regulation of energy balance. Cell 2001;104:531-43

21 Dhillo WS, Bloom SR. Hypothalamic peptides as drug targets for obesity. Curr Opin Pharmacol 2001;1:651-5.

22 Marx J. Cellular warriors at the battle of the bulge. Science 2003;299:846-9.

23 Asakawa A, Invi A, Kaga T, et al. Ghrelin is an appetite-stimulatory signal from stomach with structural resemblance to motilin. Gastroenterology $2001 ; 120: 337-45$

24 Nakazato M, Murakami N, Date Y, et al. A role for ghrelin in the central regulation of feeding. Nature 2001;409:194-8.

25 Toshinai K, Date Y, Murakami N, et al. Ghrelin-induced food intake is mediated via the orexin pathway. Endocrinology 2003;144:1506-12.

26 Duggan JP, Booth DA. Obesity, overeating, and rapid gastric emptying in rats with ventromedial hypothalamic lesions. Science 1986;231:609-11.

27 Invi A. Cancer anorexia-cachexia syndrome: are neuropeptides the key? Cancer Res 1999;59:4493-501.

28 Invi A. Cancer anorexia-cachexia syndrome: Current issues in research and management. CA-Cancer J Clin 2002;52:72-91.

29 Baldanzi G, Filigheddu N, Cutrupi S, et al. Ghrelin and des-acyl ghrelin inhibit cell death in cardiomyocytes and endothelial cells through ERK1 $/ 2$ and PI 3-kinase/AKT. J Cell Biol 2002;159:1029-37.

30 Bedendi I, Alloatti G, Marcantoni A, et al. Cardiac effects of ghrelin and its endogenous derivatives des-octanoyl ghrelin and des-Gln 14-ghrelin. Eur J Pharmacol 2003;476:87-95.

31 Asakawa A, Invi A, Kaga T, et al. Antagonism of ghrelin receptor reduces food intake and body weight gain in mice. Gut 2003;52:947-52.

32 Invi A, Asakawa A, Bowers CY, et al. Ghrelin, appetite, and gastric motility: the emerging role of the stomach as an endocrine organ. FASEB $J$ 2004; 18:439-56

33 Lawrence CB, Snape AC, Baudoin FM, et al. Acute central ghrelin and GH secretagogues induce feeding and activate brain appetite centers. Endocrinology 2002;143:155-62.

34 Elias CF, Lee CE, Kelly JF, et al. Characterization of CART neurons in the rat and human hypothalamus. J Comp Neurol 2001;432:1-19.

35 Skelton KH, Owens MJ, Nemeroff CB. The neurobiology of urocortin. Regul Pept 2000;93:85-92

36 Date Y, Murakami N, Toshinai K, et al. The role of the gastric afferent vagal nerve in ghrelin-induced feeding and growth hormone secretion in rats. Gastroenterology 2002;123:1120-8.

37 Banks WA, Tschop M, Robinson SM, et al. Extent and direction of ghrelin transport across the blood-brain barrier is determined by its unique primary structure. J Pharmacol Exp Ther 2002;302:822-7.

38 Thompson NM, Gill DA, Davies R, et al. Ghrelin and des-octanoyl ghrelin promote adipogenesis directly in vivo by a mechanism independent of the type la growth hormone secretagogue receptor. Endocrinology 2004:145:234-42.

39 Zhang W, Zhao L, Lin TR, et al. Inhibition of adipogenesis by ghrelin. Mol Biol Cell 2004;15:2484-91. 